\title{
Effects of active-sterile neutrino mixing during primordial nucleosynthesis
}

\author{
Osvaldo Civitarese \\ Department of Physics, University of La Plata, \\ 49 y 115. c.c. 67 (1900), La Plata, Argentina \\ osvaldo.civitarese@fisica.unlp.edu.ar \\ Mercedes Elisa Mosquera* and María Manuela Sáez \\ Facultad de Ciencias Astronómicas y Geofísicas, \\ Universidad Nacional de La Plata, \\ Paseo del Bosque, (1900) La Plata, Argentina \\ *mmosquera@fcaglp.unlp.edu.ar
}

Received 21 October 2014

Accepted 24 October 2014

Published 24 November 2014

\begin{abstract}
In the present work, we discuss the effects of the inclusion of sterile-active neutrino oscillations during the production of primordial light-nuclei. We assume that the sterile neutrino mass-eigenstate might oscillate with the two lightest active neutrino masseigenstates, with mixing angles $\phi_{1}$ and $\phi_{2}$. We also allow a constant renormalization (represented by a parameter $(\zeta)$ ) of the sterile neutrino occupation factor. Taking $\zeta$ and the mixing angles as free parameters, we have computed distribution functions of active and sterile neutrinos and primordial abundances. Using observable data we set constrains in the free parameters of the model. It is found that the data on primordial abundances are consistent with small mixing angles and with a value of $\zeta$ smaller than 0.65 at $3 \sigma$ level.
\end{abstract}

Keywords: Sterile neutrino; neutrino mixing; primordial nucleosysthesis.

PACS Number(s): 98.80.-k, 14.60.Pq, 26.35.+c

\section{Introduction}

The ${ }^{7} \mathrm{Li}$ problem comes from the comparison between the observational data and the theoretical results for the primordial abundance of lithium when the value of the baryon density, obtained from the analysis of the data from the Wilkinson Microwave Anisotropy Probe (WMAP) and from Planck, ${ }^{1,2}$ is used. There exist several attempts to solve this problem, such as a better understanding of turbulent transport in the radiative zone of stars, ${ }^{3}$ the existence of a stellar lithium depletion, ${ }^{4,5}$ and the inclusion of resonances in reaction rates activated in the big bang 
nucleosynthesis (BBN) decay path. ${ }^{6-8}$ Despite the efforts, the problem persists and the observed abundance of primordial lithium is smaller than the predicted BBN abundance.

Neutrino flavor oscillations have been observed ${ }^{9-16}$ and described in terms of the mixing between three active neutrino mass eigenstates, which constitutes the basis for each flavor. The results of the Liquid Scintillator Neutrino Detector (LSND) ${ }^{17}$ and the Mini Booster Neutrino Experiment (MiniBooNE) ${ }^{18}$ indicate the occurrence of some anomalies which may be interpreted as possible signals of extra neutrino species.

The effects of active-sterile neutrino oscillations during primordial nucleosynthesis have been analyzed by several authors. ${ }^{19-22}$ In particular, in Refs. 23-25 the effect of a neutrino asymmetry was studied. Other authors ${ }^{26-28}$ have studied consequences of assuming full and/or partial thermalization of the sterile neutrino distribution during BBN. The active-sterile neutrino oscillations affect the primordial abundances of light nuclei through the modification of the beta decay rates.

In previous works, we have focused on the effect of active-sterile neutrino oscillations during the epoch of light nuclei production. In Refs. 29-31 we have discussed the effects of active-sterile neutrino oscillations in different $n+1$ schemes $(n$ active neutrinos, one sterile neutrino). In Ref. 32 we have performed the analysis in the $3+1$ scheme, with a variable normalization of the sterile neutrino sector, and we were able to set constrains on the mixing parameters from the comparison between theoretical results and observable data.

In this work, we continue with our search for signals of sterile neutrinos in BBN processes, by including one sterile neutrino which can oscillate with two of the lightest active mass-eigenstates, and by taking a variable normalization constant $(\zeta)$ for the occupation factor of the sterile neutrino. We have solved quantum kinetic equations (QKE) numerically in order to obtain the distribution functions of active and sterile neutrinos. We have solved these equations for two different cases: (i) considering that the neutrino-antineutrino interaction is null $(C=0)$; and (ii) with $C \neq 0$, being $C$ the strength of the interaction, as it is explained in the next section. Using the available data on primordial abundances (observational data), we set constrains on the free parameters of our model, namely $\zeta$ and the two mixing angles.

This paper is organized as follows. In Sec. 2 we describe the formalism to include active-sterile neutrino oscillations in the production of light nuclei. In Sec. 3, we present and discuss the results of the calculation of primordial abundances. We have extracted allowed values of $\zeta$ and the mixing angles by performing a statistical analysis of the calculated abundances. Finally, the conclusions are drawn in Sec. 4 .

\section{Formalism}

The inclusion of a new kind of neutrino, the sterile neutrino, affects the neutrino density of active neutrinos due to active-sterile neutrino oscillation. In the $3+1$ 
scheme, there exists three mixing angles between the sterile neutrino and active neutrinos. In this work, we assume that the mixing between the sterile neutrino and the heaviest neutrino mass eigenstate is null, therefore we have considered two mixing angles, $\phi_{1}$ and $\phi_{2}$.

In order to compute the statistical occupation factors of neutrinos of a given flavor, we follow the formalism of Ref. 23. One can write neutrino densities as functions of a set of parameters $P_{i}$, which depend on the neutrino energy and on the temperature, then

$$
\begin{aligned}
& n_{\nu_{e}}=\frac{1}{2} P_{0}\left(1+P_{3}+\frac{1}{\sqrt{3}} P_{8}\right) n^{e q} \\
& n_{\nu_{\mu}}=\frac{1}{2} P_{0}\left(1-\frac{2}{\sqrt{3}} P_{8}\right) n^{e q} \\
& n_{\nu_{s}}=\frac{1}{2} P_{0}\left(1-P_{3}+\frac{1}{\sqrt{3}} P_{8}\right) n^{e q},
\end{aligned}
$$

where $n_{\nu_{e}}, n_{\nu_{\mu}}$ and $n_{\nu_{s}}$ are occupation factors for the electron, muon and sterile neutrinos, respectively, and $n^{e q}$ is the Fermi-Dirac distribution function for each type of particles. The QKE for those parameters can be written as ${ }^{33}$

$$
\begin{aligned}
\frac{d \mathbf{P}}{d t}= & \mathbf{V} \times \mathbf{P}-D\left(P_{1} \hat{x}_{1}+P_{2} \hat{x}_{2}+P_{6} \hat{x}_{6}+P_{7} \hat{x}_{7}\right)-D^{\prime}\left(P_{4} \hat{x}_{4}+P_{5} \hat{x}_{5}\right) \\
& -C\left(\bar{P}_{4} \hat{x}_{4}-\bar{P}_{5} \hat{x}_{4}\right)+\frac{2}{3}\left[\left(\frac{3}{2}-P_{3}\right) \frac{R_{e}}{P_{0}}-P_{3} \frac{R_{\mu}}{P_{0}}\right] \hat{x}_{3} \\
& -\left(\frac{P_{1}}{P_{0}} \hat{x}_{1}+\frac{P_{2}}{P_{0}} \hat{x}_{2}+\frac{P_{4}}{P_{0}} \hat{x}_{4}+\frac{P_{5}}{P_{0}} \hat{x}_{5}+\frac{P_{6}}{P_{0}} \hat{x}_{6}+\frac{P_{7}}{P_{0}} \hat{x}_{7}\right) \frac{d P_{0}}{d t} \\
& +\frac{2}{3}\left[\left(\frac{\sqrt{3}}{2}-P_{8}\right) \frac{R_{e}}{P_{0}}-\left(\sqrt{3}+P_{8}\right) \frac{R_{\mu}}{P_{0}}\right] \hat{x}_{8} \\
& +\left(-P_{6} \operatorname{Re}(H)-P_{7} \operatorname{Im}(H)\right) \hat{x}_{1}+\left(-P_{6} \operatorname{Im}(H)+P_{7} \operatorname{Re}(H)\right) \hat{x}_{2} \\
& +\left(-P_{1} \operatorname{Re}(H)-P_{2} \operatorname{Im}(H)\right) \hat{x}_{6}+\left(-P_{1} \operatorname{Im}(H)+P_{2} \operatorname{Re}(H)\right) \hat{x}_{7} \\
\frac{d P_{0}}{d t}= & \frac{2}{3}\left(R_{e}+R_{\mu}\right) .
\end{aligned}
$$

In the previous equation, $\mathbf{P}$ is the vector with components $P_{i}$ and $\mathbf{V}$ is the effective potential

$$
\begin{aligned}
\mathbf{V}= & 2 \operatorname{Re}\left(E^{e s}\right) \hat{x}_{1}-2 \operatorname{Im}\left(E^{e s}\right) \hat{x}_{2}+\left(E^{e e}-E^{s s}\right) \hat{x}_{3}+2 \operatorname{Re}\left(E^{e \mu}\right) \hat{x}_{4}-2 \operatorname{Im}\left(E^{e \mu}\right) \hat{x}_{5} \\
& +2 \operatorname{Re}\left(E^{s \mu}\right) \hat{x}_{6}-2 \operatorname{Im}\left(E^{s \mu}\right) \hat{x}_{7}+\frac{1}{\sqrt{3}}\left(E^{e e}+E^{s s}-2 E^{\mu \mu}\right) \hat{x}_{8},
\end{aligned}
$$

where

$$
E^{\alpha \beta}=\left[\frac{1}{2 p} U \operatorname{diag}\left(m_{1}^{2}, m_{2}^{2}, m_{3}^{2}\right) U^{\dagger}\right]^{\alpha \beta}+V^{\alpha \beta}
$$


$m_{1}, m_{2}$ and $m_{3}$ are the masses of the mass eigenstates, $p$ is the neutrino momentum, and $U$ is the mixing matrix

$$
U=\left(\begin{array}{ccc}
c_{1} c-s_{1} s_{2} s & s_{1} c_{2} & c_{1} s+s_{1} s_{2} c \\
-s_{1} c-c_{1} s_{2} s & c_{1} c_{2} & s_{1} s+c_{1} s_{2} c \\
-c_{2} s & -s_{2} & c_{2} c
\end{array}\right) .
$$

In this notation $s_{i}$ stands for $\sin \phi_{i}, c_{i}=\cos \phi_{i}, s=\sin \theta$ and $c=\cos \theta$, where $\theta$ is the mixing angle between the two active-neutrino mass eigenstates. The diagonal terms of the neutrino interaction are written as

$$
V^{\alpha \alpha}=\frac{4 \zeta(3) \sqrt{2} G_{F} T^{3}}{2 \pi^{2}}\left[L^{\alpha}+A_{\alpha} \frac{T p}{M_{W}^{2}}\right],
$$

where $G_{F}$ is the Fermi constant, $T$ stands for the temperatures, $\zeta(3)$ is a Reimann zeta-function, $M_{W}$ is the $W$-boson mass and $L^{\alpha}$ is the lepton asymmetry. The values for the constants $A_{\alpha}$ are $A_{e} \simeq 17$ and $A_{\mu, \tau} \simeq 4.9 .{ }^{23}$ The nondiagonal terms of the potential are neglected, as well as the lepton asymmetry $\left(L^{\alpha}=0\right)$.

The quantities $D$ and $D^{\prime}$ of Eq. (2) are damping parameters

$$
\begin{aligned}
D & =\frac{1}{2} G_{F}^{2} T^{5} y_{e} \frac{p}{\left\langle p_{0}\right\rangle} \\
D^{\prime} & =\frac{1}{2} G_{F}^{2} T^{5} y_{\mu} \frac{p}{\left\langle p_{0}\right\rangle},
\end{aligned}
$$

where $y_{e}=4, y_{\mu}=2.9$ and $\left\langle p_{0}\right\rangle$ is the averaged momentum for relativistic FermiDirac distribution with zero chemical potential. ${ }^{23}$ The parameter $C$ is the strength of the coupling between the neutrino and antineutrino density matrices, and it can be written as

$$
C=1.8 G_{F}^{2} T^{5}
$$

In Eq. (2) $R_{\alpha}$ are re-population functions, and $H$ are exchange factors ${ }^{23}$ which are smaller than the damping functions $D$ and $D^{\prime} .{ }^{23}$ To solve these 16 coupled differential equations (the unknown are the eight factors $P_{i}$ for neutrinos and the eight factors for anti-neutrinos), we have assumed that the factor $P_{0}$ is constant (due to the fact that the re-population factors are small), and we have neglected the function $H$ and the nondiagonal terms on the neutrino potential, as said before. We have performed the calculations for two different cases: (i) by considering that the coupling between neutrino and anti-neutrino density matrices is null $(C=0)$, and (ii) taking $C \neq 0$.

The initial condition was set at $T_{0}=3 \mathrm{MeV}$, and we have assumed that the active neutrinos have standard Fermi-Dirac distributions at that temperature. For the sterile neutrino we have considered two different situations for its occupation factor, namely: (i) a null occupation factor; and (ii) a Fermi-Dirac distribution multiplied by a constant factor $\zeta$ (Refs. 34 and 35) which can vary from 0 to 1 .

With these parameters and approximations, we have calculated primordial abundances by using a modified version of the Kawano's code ${ }^{36,37}$ (see Ref. 31 
for more details on the modifications to the standard code). In the calculations, the value of the square-mass difference (between the lightest active neutrino mass and the sterile neutrino mass) was fixed at $1 \mathrm{eV}^{2} \cdot 38,39$

\section{Results}

The value of the active-neutrino mixing, namely the angle $\theta$ and the square-mass difference, have been determined from SNO, SK, GNO, CHOOZ DAYA BAY, RENO and DOUBLE CHOOZ experiments. ${ }^{9,14,15,40-43}$ The baryon density was fixed at the value determined by WMAP Collaboration. ${ }^{1}$

The observational data for deuterium (D) have been extracted from Refs. 44-50. We use the data from Refs. $51-58$ for ${ }^{4} \mathrm{He}$, and for ${ }^{7} \mathrm{Li}$ we have considered the data given by Refs. 59-62. Regarding the consistency of the data, we have followed the treatment of Ref. 63 .

\subsection{Results with $C=0$}

As a first case, we have computed the primordial abundance of light nuclei for different values for the active-sterile neutrino mixing at a fixed baryon density and a null initial condition for the sterile neutrino (meaning $\zeta=0$ ). In order to obtain the best values for the parameters, $\sin ^{2} 2 \phi_{1}$ and $\sin ^{2} 2 \phi_{2}$, we have performed a $\chi^{2}$-test. Results are presented in the first two rows of Table 1.

The $\chi^{2}$-test indicates that the global fit is not a good one if the complete set of data is included in the analysis, however, a better fit is obtained if the data on primordial lithium are removed from the sample of observational data. The best values for the mixing angles are small in both cases and consistent with zero at $2 \sigma$-level.

Next, we have considered the parameter $\zeta$ as an extra parameter to adjust. The results of the statistical analysis is presented in the third row of Table 1. Once again, the statistical analysis does not give a good fit, the values for the mixing angles remain small and consistent with a null mixing angle, meanwhile the parameter $\zeta$ increases its value.

\subsection{Results with $C \neq 0$}

The next step in the analysis was to turn-on the interaction between neutrinos and anti-neutrinos by setting $C \neq 0$. Once again, we have computed neutrino occupation

Table 1. Best-fit parameter values and $1 \sigma$ errors (case $C=0$ ).

\begin{tabular}{lcccr}
\hline Data & $\zeta \pm \sigma$ & $\sin ^{2} 2 \phi_{1} \pm \sigma$ & $\sin ^{2} 2 \phi_{2} \pm \sigma$ & $\frac{\chi^{2}}{N-a}$ \\
\hline $\mathrm{D}+{ }^{4} \mathrm{He}+{ }^{7} \mathrm{Li}$ & fixed at 0 & $0.019^{+0.026}$ & $0.076_{-0.055}^{+0.058}$ & 9.82 \\
$\mathrm{D}+{ }^{4} \mathrm{He}$ & fixed at 0 & $0.022^{+0.027}$ & $0.093_{-0.059}^{+0.063}$ & 1.04 \\
$\mathrm{D}+{ }^{4} \mathrm{He}+{ }^{7} \mathrm{Li}$ & $0.30 \pm 0.12$ & $0.025^{+0.012}$ & $0.000^{+0.093}$ & 10.04 \\
\hline
\end{tabular}


Table 2. Best-fit parameter values and $1 \sigma$ errors considering $C \neq 0$.

\begin{tabular}{lcccr}
\hline Data & $\zeta \pm \sigma$ & $\sin ^{2} 2 \phi_{1} \pm \sigma$ & $\sin ^{2} 2 \phi_{2} \pm \sigma$ & $\frac{\chi^{2}}{N-a}$ \\
\hline $\mathrm{D}+{ }^{4} \mathrm{He}+{ }^{7} \mathrm{Li}$ & fixed at 0 & $0.002^{+0.007}$ & $0.040 \pm 0.033$ & 9.82 \\
$\mathrm{D}+{ }^{4} \mathrm{He}$ & fixed at 0 & $0.007^{+0.004}$ & $0.020_{-0.009}^{+0.078}$ & 1.04 \\
$\mathrm{D}+{ }^{4} \mathrm{He}+{ }^{7} \mathrm{Li}$ & $0.30_{-0.13}^{+0.11}$ & $0.000^{+0.004}$ & $0.014^{+0.023}$ & 10.04 \\
\hline
\end{tabular}

factors and primordial abundances and compared them to the observational data through a statistical test. The results, with a fixed value of the parameter $\zeta$ are shown in the first two rows of Table 2 .

Also for this case, there is not a good fit when all data are used in the analysis, but it improves if one removes the data on ${ }^{7} \mathrm{Li}$. The mixing angles are smaller that the ones obtained in the previous section (case $C=0$ ).

As a final analysis, we have adjusted the renormalization parameter $\zeta$. The fit is not a good one, however, the value for $\zeta$ is in agreement with the one obtained previously, and the mixing angles remain small and consistent with a null value.

\section{Conclusion}

In this work, we have included a sterile neutrino in the formalism of primordial nucleosynthesis and computed the abundances of the light nuclei as a function of the mixing parameters. We have computed neutrino occupation factors and neutron-toproton decay rates, as functions of the introduced mixing parameters, and extracted their values from the comparison between calculated and observed primordial abundances. We have found that the two added active-sterile mixing angles are small and that they are consistent with zero at $1 \sigma$ or $2 \sigma$. The value of the parameter $\zeta$, which is the renormalization factor of the sterile neutrino thermal occupation, is found to be the same for the two cases considered in this work (neutrino-antineutrino density coupling $C=0$ and $C \neq 0$ ). The results are found to be consistent with previous works. ${ }^{20,29-32}$

\section{Acknowledgments}

The support for this work was provided by the National Research Council (CONICET) of Argentina, and by the ANPCYT of Argentina. O. C. and M. E. M. are members of the Scientific Research Career of the CONICET.

\section{References}

1. G. Hinshaw et al., Astrophys. J. Suppl. 208 (2013) 19.

2. Planck Collab. (P. A. R. Ade et al.), arXiv:1303.5076.

3. O. Richard, G. Michaud and J. Richer, Astrophys. J. 619 (2005) 538.

4. J. Meléndez et al., Astron. Astrophys. 515 (2010) L3.

5. K. Lind et al., in IAU Symposium, eds. C. Charbonnel, M. Tosi, F. Primas and C. Chiappini, Vol. 268 (IAU Symposium, 2010), pp. 263-268. 
6. O. S. Kirsebom and B. Davids, Phys. Rev. C 84 (2011) 058801.

7. C. Broggini, L. Canton, G. Fiorentini and F. L. Villante, arXiv:1202.5232.

8. O. Civitarese and M. E. Mosquera, Nucl. Phys. A 898 (2013) 1.

9. CHOOZ Collab. (M. Apollonio et al.), Phys. Lett. B 466 (1999) 415.

10. SAGE Collab. (J. N. Abdurashitov et al.), Phys. Rev. C 80 (2009) 015807.

11. SNO Collab. (B. Aharmim et al.), Phys. Rev. C 87 (2013) 015502.

12. Super-Kamiokande Collab. (K. Abe et al.), Phys. Rev. D 83 (2011) 052010.

13. Kamland Collab. (A. Gando et al.), Phys. Rev. D 83 (2011) 052002.

14. GNO Collab. (M. Altmann et al.), Phys. Lett. B 616 (2005) 174.

15. K2K Collab. (M. H. Ahn et al.), Phys. Rev. D 74 (2006) 072003.

16. Borexino Collab. (C. Arpesella et al.), Phys. Rev. Lett. 101 (2008) 091302.

17. LSND Collab. (A. Aguilar et al.), Phys. Rev. D 64 (2001) 112007.

18. MiniBooNE Collab. (A. A. Aguilar-Arevalo et al.), Phys. Rev. Lett. 110 (2013) 161801.

19. C. J. Smith, G. M. Fuller, C. T. Kishimoto and K. N. Abazajian, Phys. Rev. D 74 (2006) 085008.

20. C. T. Kishimoto, G. M. Fuller and C. J. Smith, Phys. Rev. Lett. 97 (2006) 141301.

21. K. Abazajian, N. F. Bell, G. M. Fuller and Y. Y. Y. Wong, Phys. Rev. D 72 (2005) 063004 .

22. P. Keraäen, J. Maalampi, M. Myyryläinen and J. Riitinen, Phys. Lett. B 574 (2003) 162.

23. N. F. Bell, R. R. Volkas and Y. Y. Y. Wong, Phys. Rev. D 59 (1999) 113001.

24. P. Di Bari, Phys. Rev. D 65 (2002) 043509.

25. R. Foot, M. J. Thomson and R. R. Volkas, Phys. Rev. D 53 (1996) R5349.

26. S. Hannestad, I. Tamborra and T. Tram, J. Cosmol. Astropart. Phys. 07 (2012) 025.

27. I. Tamborra, S. Hannestad and T. Tram, Nucl. Phys. B (Proc. Suppl.) 237-238 (2013) 256.

28. T. D. Jacques, L. M. Krauss and C. Lunardini, Phys. Rev. D 87 (2013) 083515.

29. O. Civitarese and M. E. Mosquera, Phys. Rev. C 77 (2008) 045806.

30. O. Civitarese and M. E. Mosquera, Int. J. Mod. Phys. E 17 (2008) 351.

31. M. E. Mosquera and O. Civitarese, Phys. Rev. C 84 (2011) 065803.

32. M. E. Mosquera and O. Civitarese, Int. J. Mod. Phys. E 23 (2014) 1450014.

33. B. H. J. MacKellar and M. J. Thomson, Phys. Rev. D 49 (1994) 2710.

34. K. Ichikawa, T. Sekiguchi and T. Takahashi, Phys. Rev. D 78 (2008) 083526.

35. M. A. Acero and J. Lesgourgues, Phys. Rev. D 79 (2009) 045026.

36. L. Kawano (1988), fERMILAB-PUB-88-034-A.

37. L. Kawano (1992), fERMILAB-PUB-92-004-A.

38. J. Kopp et al., J. High Energy Phys. 5 (2013) 50.

39. M. Archidiacono et al., Phys. Rev. D 87 (2013) 125034.

40. Daya Bay Collab. (F. P. An et al.), Phys. Rev. Lett. 108 (2012) 171803.

41. RENO Collab. (Soo-Bong Kim et al.), Phys. Rev. Lett. 108 (2012) 191802.

42. Double Chooz Collab. (Y. Abe et al.), Phys.Lett. B 723 (2013) 66.

43. SNO Collab. (B. Aharmim et al.), Phys. Rev. C 88 (2013) 025501.

44. P. Noterdaeme et al., Astron. Astrophys. 542 (2012) L33.

45. M. Pettini et al., Mon. Not. R. Astron. Soc. 391 (2008) 1499.

46. S. A. Balashev, A. V. Ivanchik and D. A. Varshalovich, Astron. Lett. 36 (2010) 761.

47. M. Pettini and R. Cooke, Mon. Not. R. Astron. Soc. 425 (2012) 2477.

48. R. J. Cooke et al., Astrophys. J. 781 (2014) 31.

49. M. Fumagalli, J. M. O'Meara and J. X. Prochaska, Science 334 (2011) 1245.

50. J. M. O'Meara et al., Astrophys. J. 649 (2006) L61. 
51. S. Villanova et al., Astrophys. J. 748 (2012) 62.

52. M. Peimbert, V. Luridiana and A. Peimbert, Astrophys. J. 666 (2007) 636.

53. Y. I. Izotov and T. X. Thuan, Astrophys. J. Lett. 710 (2010) L67.

54. E. Aver, K. A. Olive and E. D. Skillman, J. Cosmol. Astropart. Phys. 04 (2012) 004.

55. S. Villanova, G. Piotto and R. G. Gratton, Astron. Astrophys. 499 (2009) 755.

56. E. Aver et al., J. Cosmol. Astropart. Phys. 11 (2013) 017.

57. Y. I. Izotov, G. Stasińska and N. G. Guseva, Astron. Astrophys. 558 (2013) A57.

58. E. Aver, K. A. Olive and E. D. Skillman, J. Cosmol. Astropart. Phys. 05 (2010) 003.

59. L. Monaco et al., Astron. Astrophys. 539 (2012) A157.

60. L. Sbordone et al., Astron. Astrophys. 522 (2010) A26.

61. K. Lind et al., Astron. Astrophys. 503 (2009) 545.

62. A. Mucciarelli, M. Salaris and P. Bonifacio, Mem. S. A. It. Suppl. 22 (2012) 86.

63. K. Nakamura et al., J. Phys. G 37 (2010) 075021. 